\title{
Nonlinear Reduced-Order Simulation Using An Experimentally Guided Modal Basis
}

\author{
Stephen A. Rizzi ${ }^{1}$ \\ NASA Langley Research Center, Hampton, VA, 23681 \\ and \\ Adam Przekop ${ }^{2}$ \\ Analytical Services and Materials, Inc., Hampton, VA, 23666
}

\begin{abstract}
A procedure is developed for using nonlinear experimental response data to guide the modal basis selection in a nonlinear reduced-order simulation. The procedure entails using nonlinear acceleration response data to first identify proper orthogonal modes. Special consideration is given to cases in which some of the desired response data is unavailable. Bases consisting of linear normal modes are then selected to best represent the experimentally determined transverse proper orthogonal modes and either experimentally determined in-plane proper orthogonal modes or the special case of numerically computed in-plane companions. The bases are subsequently used in nonlinear modal reduction and dynamic response simulations. The experimental data used in this work is simulated to allow some practical considerations, such as the availability of in-plane response data and non-idealized test conditions, to be explored. Comparisons of the nonlinear reduced-order simulations are made with the surrogate experimental data to demonstrate the effectiveness of the approach.
\end{abstract}

\section{Nomenclature}

$\mathbf{C}, \tilde{\mathbf{C}} \quad=$ system damping matrix in physical and modal degrees of freedom

f, $\tilde{\mathbf{f}} \quad=$ force excitation vector in physical and modal degrees of freedom

$\mathbf{f}_{\mathrm{NL}}, \tilde{\mathbf{f}}_{\mathrm{NL}}=$ nonlinear restoring force vector in physical and modal degrees of freedom

$\Phi=$ modal basis matrix

$\lambda=$ proper orthogonal value (POV) matrix

$\mathbf{M}, \tilde{\mathbf{M}}=$ system mass matrix in physical and modal degrees of freedom

$v=$ cumulative modal amplitude participation factor

$\omega \quad=$ undamped natural frequency

$\mathbf{P}=$ proper orthogonal mode $(\mathrm{POM})$ matrix

$\mathbf{R}_{\mathrm{X}} \quad=$ displacement correlation matrix

$\mathbf{x}, \mathbf{q}=$ displacement response vector in physical and modal degrees of freedom

$\mathbf{X}=$ displacement snapshot matrix

$\chi \quad=$ modal amplitude participation factor

$\zeta=$ viscous damping factor

\section{Introduction}

JONLINEAR random response analysis of large structural components in physical degrees of freedom (DoFs) I can be associated with a prohibitive computational cost. Reduced-order modeling has been shown to be a viable alternative for a range of loading and response conditions. ${ }^{1-8}$ For a typical aerospace structure having

\footnotetext{
${ }^{1}$ Senior Research Engineer, Structural Acoustics Branch, Associate Fellow AIAA.

${ }^{2}$ Senior Structural Engineer, Senior Member AIAA.
} 
complex geometry and loading conditions (combined mechanical, thermal, acoustic, and pressure), selection of basis functions through which the reduced-order system is formed is usually not intuitive. A rigorous modal basis selection procedure resulting in a computationally efficient and accurate reduced-order simulation was recently developed. ${ }^{9}$ As part of that procedure, system identification was performed and a criterion for modal basis selection was established. In that work, the system identification procedure used nonlinear dynamic response data from a fullorder numerical simulation in physical DoFs. The data consisted of both transverse and in-plane DoFs. The goal of this work is to explore the use of experimental data in the system identification procedure. A key benefit of the approach will be the elimination of a full-order simulation currently required for the system identification procedure.

An aluminum beam structure under a transverse, point-load, random excitation is used to demonstrate the approach. Two variations are considered: an idealized case with perfectly clamped boundary conditions and known transducer mass and location, and a non-idealized case with unknown compliant boundary conditions and perturbed transducer mass and locations. For the idealized case, nonlinear acceleration response data is used in a proper orthogonal decomposition (POD) analysis to obtain proper orthogonal modes (POMs). Since in-plane acceleration data is not typically measured, transverse acceleration data are used to identify only the transverse POMs. These, in turn, are used as static displacement fields in a nonlinear static analysis to determine corresponding in-plane behavior. A load-independent modal basis is subsequently identified consisting of a set of linear normal modes (LNMs) resembling the combination of experimentally determined transverse POMs and numerically determined inplane displacement shapes. An alternative approach, using experimentally determined in-plane POMs instead of numerically determined displacement shapes, is also demonstrated. For the non-idealized case, the linear dynamics model is first updated to match the linear vibration response. The model update procedure uses linear static deflection data to adjust the stiffness, and the fundamental frequency to adjust the mass. The basis selection considered uses experimentally determined transverse POMs and numerically determined in-plane displacement shapes. Note that the nonlinear modal reduction used by the authors does not statically condense in-plane DoFs. ${ }^{3}$ Therefore, a technique similar to the current approach, ${ }^{10}$ but which identifies only transverse LNMs, would generate only part of the required basis.

Because the focus of this work is on methods development, simulated experimental data is used in lieu of actual experimental data. This surrogate experimental data offers the ability to explore practical considerations including the availability of in-plane acceleration data and the effect of modeling uncertainty on the simulated response. Further, it serves to guide further experimental studies insofar as measurement specifications are concerned.

\section{Nonlinear Modal Reduction and Simulation}

Since the modal reduction utilizing the indirect approach employing a nonlinear stiffness evaluation procedure was detailed by the authors in several recent publications, ${ }^{1,11-14}$ only an abbreviated description is presented herein. The equations of motion of the nonlinear full-order system in physical DoFs may be written as

$$
\mathbf{M} \ddot{\mathbf{x}}(t)+\mathbf{C} \dot{\mathbf{x}}(t)+\mathbf{f}_{\mathrm{NL}}(\mathbf{x}(t))=\mathbf{f}(t)
$$

where $\mathbf{M}$ and $\mathbf{C}$ are the system mass and damping matrices, $\mathbf{x}$ is the displacement response vector and $\mathbf{f}$ is the force excitation vector, respectively. The nonlinear restoring force $\mathbf{f}_{\mathrm{NL}}$ is a vector function, which generally includes the linear, quadratic, and cubic stiffness terms. By applying the modal coordinate transformation

$$
\mathbf{x}(t)=\Phi \mathbf{q}(t)
$$

to Eq. (1), a modal equation of motion can be written as

$$
\tilde{\mathbf{M}} \ddot{\mathbf{q}}(t)+\tilde{\mathbf{C}} \dot{\mathbf{q}}(t)+\tilde{\mathbf{f}}_{\mathrm{NL}}\left(\mathbf{q}_{1}(t), \mathbf{q}_{2}(t), \ldots, \mathbf{q}_{L}(t)\right)=\tilde{\mathbf{f}}(t)
$$

where $\mathbf{q}=\left[q_{1}, q_{2}, \ldots, q_{L}\right]^{\mathrm{T}}$ is a generalized coordinate vector and $\boldsymbol{\Phi}$ is the set of $L$ selected LNMs. For massnormalized eigenvectors,

$$
\tilde{\mathbf{M}}=\boldsymbol{\Phi}^{\mathrm{T}} \mathbf{M} \boldsymbol{\Phi}=\lceil\mathbf{I}\rfloor \quad \tilde{\mathbf{C}}=\boldsymbol{\Phi}^{\mathrm{T}} \mathbf{C} \boldsymbol{\Phi}=\left\lceil 2 \zeta_{r} \omega_{r}\right\rfloor
$$


and $\omega_{r}$ are the undamped natural frequencies and $\zeta_{r}$ are the viscous damping factors. The modal excitation force is $\tilde{\mathbf{f}}=\boldsymbol{\Phi}^{\mathrm{T}} \mathbf{f}$.

Since the nonlinear restoring force $\mathbf{f}_{\mathrm{NL}}$ is generally not known in the context of a commercial finite element (FE) program, an indirect means of evaluating the nonlinear stiffness is required. Different combinations of scaled LNMs can be used to form a set of prescribed displacement fields. Using a nonlinear FE static analysis, nonlinear restoring forces $\mathbf{f}_{\mathrm{NL}}$ can be computed in physical DoFs and transformed to the generalized coordinates as $\tilde{\mathbf{f}}_{\mathrm{NL}}=\boldsymbol{\Phi}^{\mathrm{T}} \mathbf{f}_{\mathrm{NL}}$. The $r^{\text {th }}$ nonlinear modal restoring force can be written as a summation of modal linear, quadratic, and cubic stiffness components as

$$
\tilde{f}_{N L}^{r}\left(q_{1}, q_{2}, \ldots, q_{L}\right)=\sum_{j=1}^{L} d_{j}^{r} q_{j}+\sum_{j=1}^{L} \sum_{k=j}^{L} a_{j k}^{r} q_{j} q_{k}+\sum_{j=1}^{L} \sum_{k=j}^{L} \sum_{l=k}^{L} b_{j k l}^{r} q_{j} q_{k} q_{l} \quad r=1,2, \ldots, L
$$

Since the left-hand-side of the Eq. (5) is known, as are the assumed scaling factors $q$ on its right-hand-side, solution of a simple algebraic system of equations is only needed to arrive at the linear $d$, quadratic $a$, and cubic $b$ modal stiffness coefficients. Eq. (3) is numerically integrated using a $4^{\text {th }}$-order Runge-Kutta scheme and physical displacements are obtained through the inverse modal coordinate transform.

\section{System Identification and Modal Basis Selection Procedure}

Three approaches were previously offered for selecting a modal basis following the system identification procedure. ${ }^{9}$ These were the modal amplitude participation (MAP), modal energy participation (MEP), and estimated POM frequency (EPF) procedures. Since the present study will consider a hybrid of experimentally determined transverse POMs and numerically determined corresponding in-plane behavior, the MAP procedure, which considers these DoF types separately, will be utilized.

When physical DoFs are chosen to characterize a response, a data set can be formed as an accumulation of $n$ instantaneous displacement fields to produce a displacement snapshot matrix $\mathbf{X}$. The snapshot matrix contains the selected set of $N$ DoFs resulting in its size being $n \times N$. The sample rate and spatial resolution of the snapshot matrix must be sufficient to resolve the system's temporal and spatial characteristics of interest. The displacement correlation matrix $\mathbf{R}_{\mathrm{X}}$ of size $N \times N$ is formed as

$$
\mathbf{R}_{\mathbf{X}}=\frac{1}{n} \mathbf{X}^{\mathrm{T}} \mathbf{X}
$$

An eigenanalysis of the displacement correlation matrix $\mathbf{R}_{\mathrm{X}}$ is next performed, i.e.,

$$
\left[\mathbf{R}_{\mathrm{x}}-\lambda \mathbf{I}\right] \mathbf{p}=\mathbf{0}
$$

to obtain the POM matrix $\mathbf{P}=\left[\begin{array}{llll}\mathbf{p}_{1} & \mathbf{p}_{2} \ldots \mathbf{p}_{N}\end{array}\right]$ and the diagonal proper orthogonal value (POV) matrix, $\lambda$, both of size $N \times N .{ }^{15}$ Note that an alternative means of obtaining similar quantities is via singular value decomposition. ${ }^{16}$

The contribution of each POM to the overall dynamic response is given by

$$
\chi_{i}=\lambda_{i} / \sum_{j=1}^{N} \lambda_{j} \quad i=1, \ldots, N
$$

where $\chi_{j}$ is the $j^{\text {th }}$ POM modal amplitude participation factor. The sum of all POM modal amplitude participation factors is unity. When the dominant $M$ POMs are selected, their cumulative participation, $v$, can be expressed as 


$$
v=\sum_{j=1}^{M} \chi_{j} \quad 0<v \leq 1
$$

Retention of only $M$ selected POMs reduces the size of $\mathbf{P}$ to $N$ x $M$. The MAP approach requires as many criteria as there are DoF types used in the system identification, e.g. one cutoff value for the transverse displacement and one value for the in-plane displacement. Using such cutoff values, a set of POMs may be selected based on the modal amplitude participation factor, the cumulative participation, or some combination thereof.

The direct use of POMs as the basis functions is not preferred as they are specific to the loading under which they were determined and, consequently, applicability of such a basis to alternative loading conditions may be limited. Instead, a more robust basis, formed from load-independent LNMs $\boldsymbol{\Phi}$ which resemble the $M$ selected POMs, is sought. The authors previously identified two means of doing so; ${ }^{11}$ one using the modal assurance criterion (MAC) ${ }^{17}$ and the other using a modal expansion. ${ }^{9,11,12}$ The MAC approach was used in this work. For a pair of POM $\mathbf{p}$ and normal mode $\varphi$ vectors, the MAC value can be obtained from

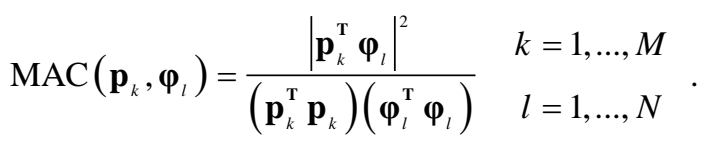

Once the MAC matrices are determined, a MAC threshold level is used to identify the normal modes best resembling the selected POMs. A threshold of MAC $\geq 0.5$ was used in this work. The end result of the basis selection process is a set of $L$ selected LNMs, $L \ll N$, for use in the nonlinear modal reduction.

\section{Idealized Case}

The idealized case was used to develop the procedure for generating transverse and in-plane POMs from acceleration data, and for generating numerically determined in-plane POM replacements in the absence of in-plane acceleration data. A planar aluminum beam structure, measuring $0.4572 \mathrm{~m}$ in length with a cross-section of $25.4 \mathrm{~mm}$ wide by $2.286 \mathrm{~mm}$ high, was previously investigated by the authors ${ }^{13}$ and was the subject of the current study. The beam was perfectly clamped at both ends and subject to transverse point-load random excitation at the 1/4-span location, as shown in Figure 1. The zero-mean Gaussian distributed excitation had a flat spectrum to $1500 \mathrm{~Hz}$. Two loading levels were considered; a low level loading of 0.1N root-mean-square (RMS) was used to excite the beam in the linear response regime, and a high level loading of 12.8N RMS was used to generate a geometrically nonlinear response.

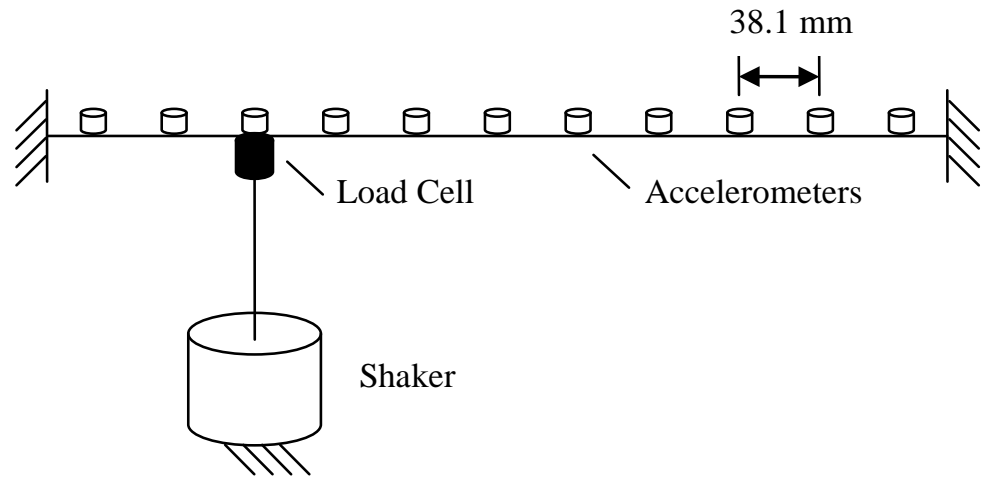

Figure 1: Schematic of virtual test setup.

\section{A. Surrogate Experiment}

As previously indicated, the "experiments" performed in this work were actually full-order (physical DoF) numerical simulations of the dynamic response under virtual test conditions. The full-order finite element model was comprised of 144 ABAQUS $^{18}$ two node B21 elements, each measuring 3.175 mm in length. The B21 element features three DoFs per node; one rotational DoF and two translational (transverse and in-plane) DoFs. Material properties were: Young's modulus $E=73.11 \mathrm{GPa}$, shear modulus $G=27.59 \mathrm{GPa}$, and mass density $\rho=2763 \mathrm{~kg} / \mathrm{m}^{3}$. 
Mass proportional damping was specified as $14.54 \mathrm{~s}^{-1}$, which corresponded to a viscous damping factor of $2.78 \%$ at the linear fundamental frequency of $41.65 \mathrm{~Hz}$.

Accelerometers were modeled as $0.4 \mathrm{~g}$ lumped masses, and were uniformly spaced $38.1 \mathrm{~mm}$ apart, as shown in Figure 1. Additionally, a 25g load cell was modeled as a lumped mass at the $1 / 4$-span drive point. The stinger and shaker shown in the schematic were not modeled. Acceleration time histories were simultaneously recorded at a sampling rate of $20 \mathrm{k}$ samples/s. Experimental data was simulated for $104 \mathrm{~s}$, with the first $0.5 \mathrm{~s}$ discarded to remove the startup transient. Transverse and in-plane acceleration power spectral densities (PSDs) at the 1/4-span drive point are shown in Figure 2 and Figure 3, respectively. A 32,768-point transform was used in these and subsequent PSDs, giving a frequency resolution of $0.61 \mathrm{~Hz}$. The nonlinear response is evident in the broad peaks and significant response outside the $0-1500 \mathrm{~Hz}$ excitation bandwidth. Note that the in-plane acceleration response rolls off at twice the excitation bandwidth (not shown) due to frequency doubling. Frequency doubling occurs because each half cycle of upward and downward transverse motion results in a full cycle of in-plane stretching.

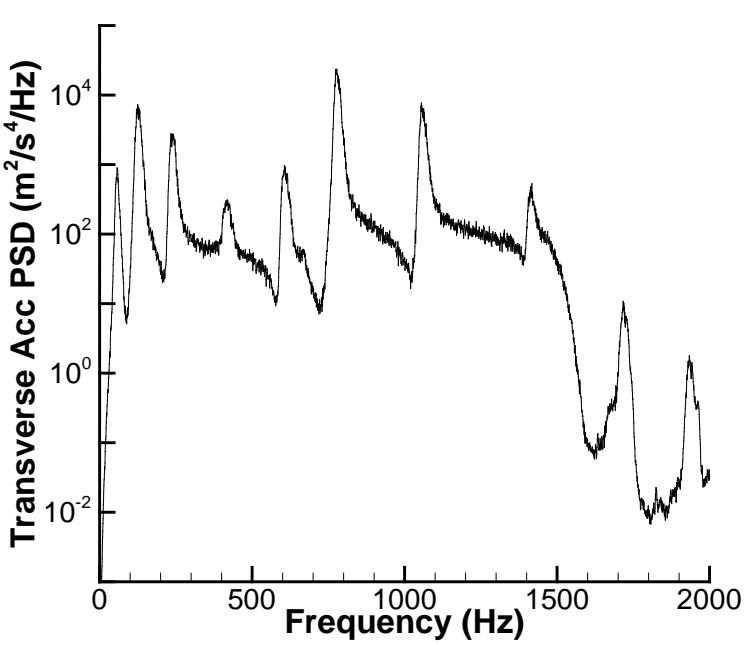

Figure 2: Nonlinear transverse drive point acceleration PSD for the 12.8N RMS loading.

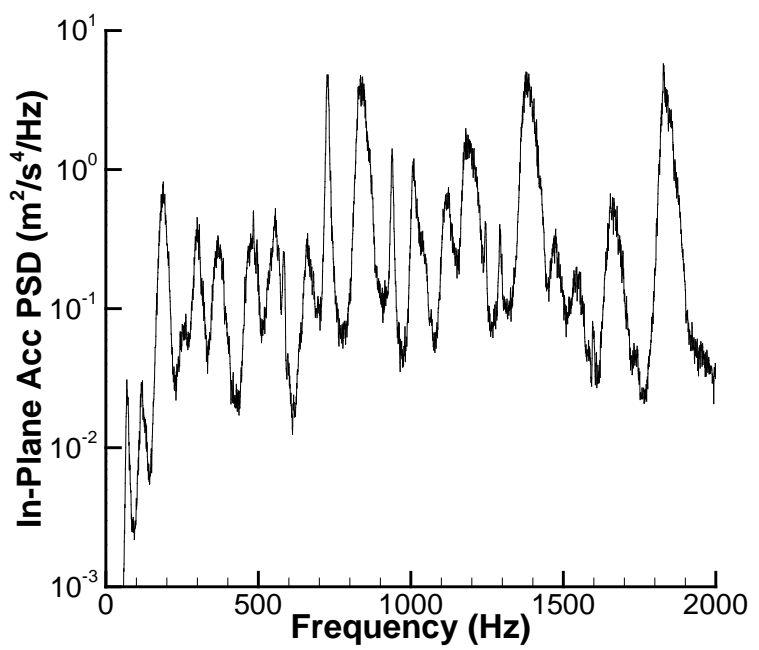

Figure 3: Nonlinear in-plane drive point acceleration PSD for the 12.8N RMS loading.

While the number of active DoFs in the finite element model is 429 (143 nodes x 3 DoF/node), the number of active DoFs in the experiment was either 11 when using uni-axial accelerometers to "measure" transverse acceleration, or 22 when using bi-axial accelerometers to "measure" both transverse and in-plane acceleration. A practical consideration in the design of the experiment therefore is that the number of measurements must be greater than or equal to the anticipated number of $M$ selected POMs.

\section{B. System Identification and Modal Basis Selection}

The POD analysis, as discussed in Section III, uses displacement time histories to form the correlation matrix, $\mathbf{R}_{\mathrm{X}}$, not the acceleration time histories. Therefore, the first step in the analysis was to integrate the nonlinear acceleration time history, obtained from the $12.8 \mathrm{~N}$ RMS loading, to obtain the displacement time history. Shorter records of duration $1.6384 \mathrm{~s}$ were used to form the 32,768-point $(n)$ displacement snapshots used in the POD analysis. The integration operation was performed in the frequency domain by taking the complex 32,768-point acceleration transform, dividing by $-\omega^{2}$, mirroring the complex conjugate about the Nyquist frequency, and taking the inverse transform to obtain the displacement. Leakage was avoided by selecting an analysis window equal to the record length, hence windowing was not required. The resulting displacement time history was then high-pass filtered with a $3^{\text {rd }}$-order Butterworth filter to remove the DC component. The MATLAB ${ }^{19}$ filter function "filtfilt" was used to eliminate phase distortion. The resulting transverse and in-plane displacement response PSDs are shown in Figure 4 and Figure 5, respectively. 


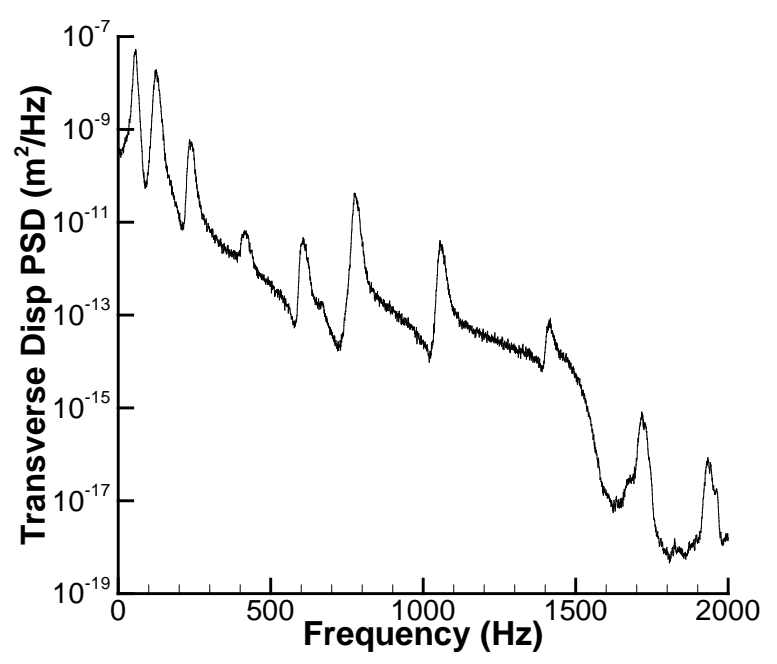

Figure 4: Nonlinear transverse drive point displacement PSD for the 12.8N RMS loading.

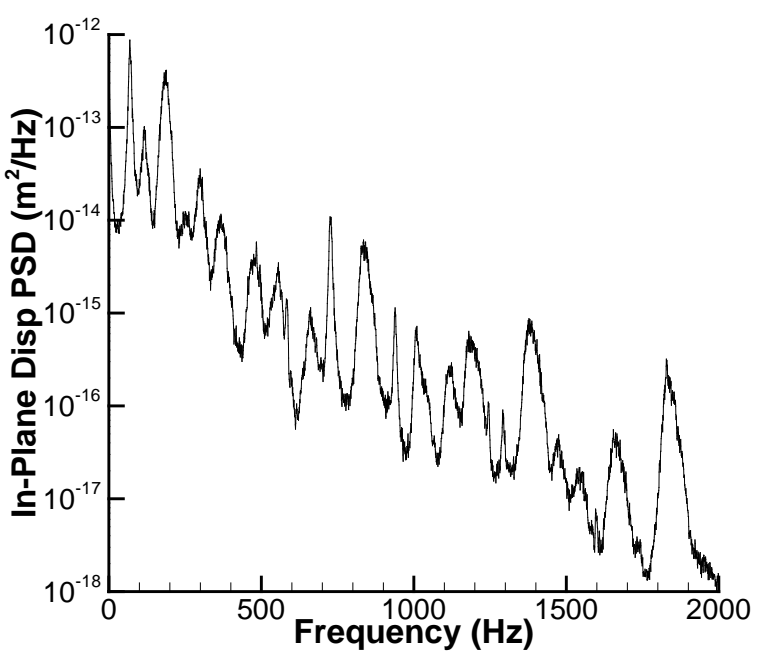

Figure 5: Nonlinear in-plane drive point displacement PSD for the 12.8N RMS loading.

\section{Transverse Modes}

In this study, transverse POMs were always determined from integrated transverse accelerometer data. These POMs served two purposes. The primary purpose was to select part of the LNM basis using the MAC analysis discussed in Section III. The second purpose was to serve as a set of prescribed transverse deflections in a nonlinear static analysis to numerically determine the corresponding in-plane displacement fields. These displacement fields act as alternatives to in-plane POMs when in-plane accelerometer data is unavailable. Because of the requirement to prescribe the transverse deflections at all the nodes and not just at the accelerometer locations, the transverse POMs were first fit with a cubic spline. Thus, another practical consideration is that an appropriate spatial distribution of accelerometer locations is required to characterize the POM shape. The spline-fit would obviously not work well, for example, if all the accelerometer locations were toward one side. For consistency, the fitting operation was performed regardless of whether or not in-plane accelerometer data was available. As such, the subsequent MAC analysis was performed using all 145 transverse DoFs $(N)$. Had the requirement to derive an in-plane POM alternative not been present, the LNM shapes obtained from the eigenanalysis of the full-order model could have been decimated to the 11 accelerometer locations.

The first 6 POMs, ranked according to their participation, as computed per Eq. (8), are shown in Figure 6 Figure 11. In each figure, the raw experimentally determined POM is shown at the nodal spacing corresponding to the accelerometer locations. The spline-fit POM used for both the MAC and nonlinear static analyses are shown at each nodal location. For comparison, POMs obtained directly from the full-order FE analysis displacement time histories are also shown. These POMs are included to contrast the present experimental approach with the former simulation-based approach. ${ }^{9,11-13}$ It is seen that the set of POMs from the two methods compare well.

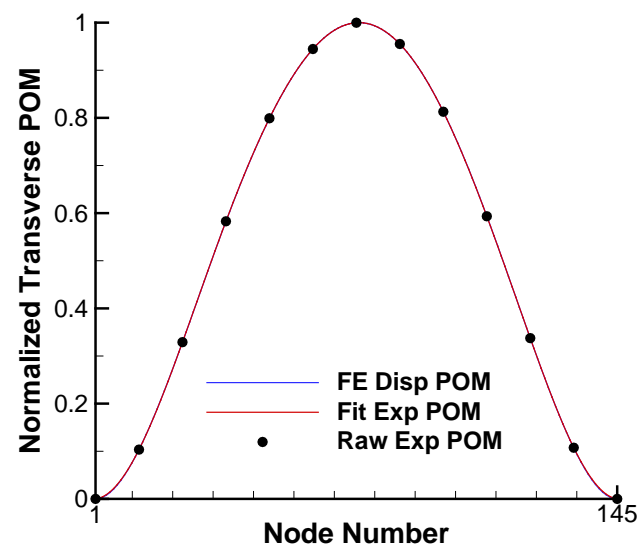

Figure 6: Transverse POM 1.

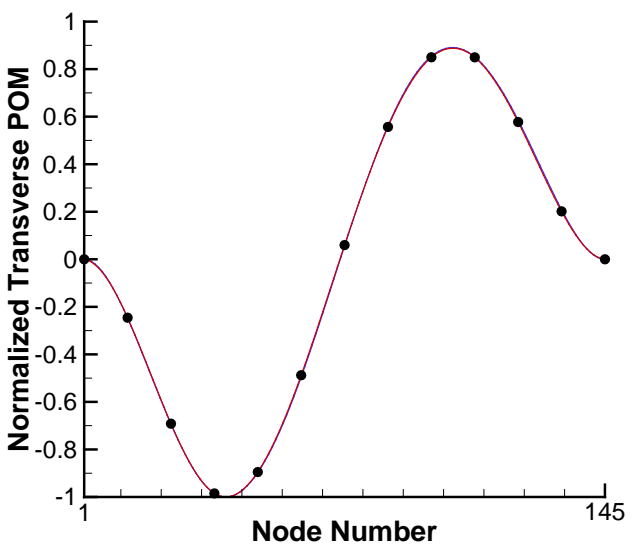

Figure 7: Transverse POM 2.

6

American Institute of Aeronautics and Astronautics 


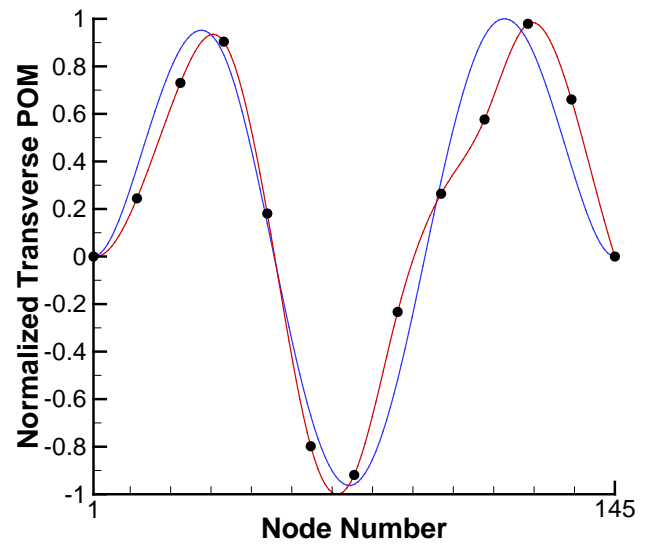

Figure 8: Transverse POM 3.

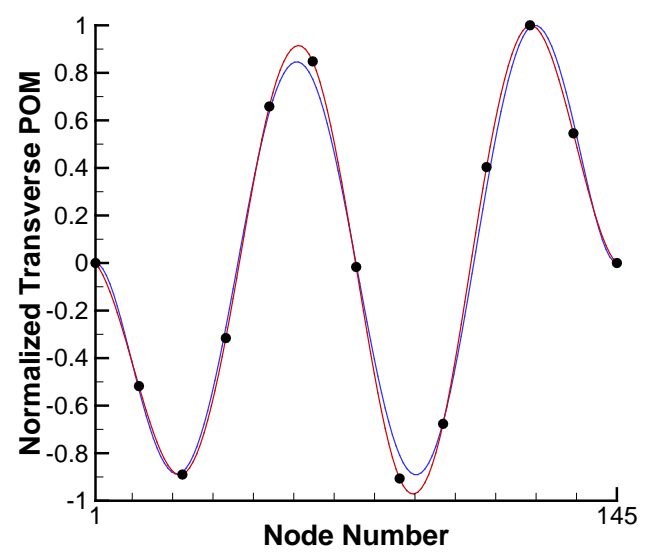

Figure 10: Transverse POM 5.

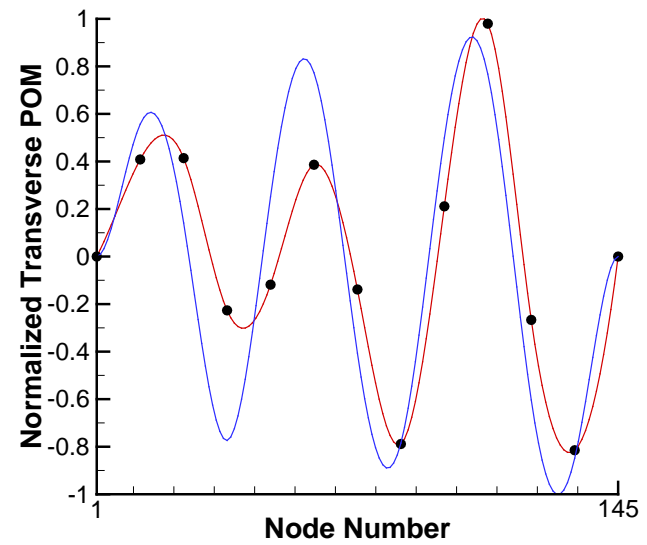

Figure 9: Transverse POM 4.

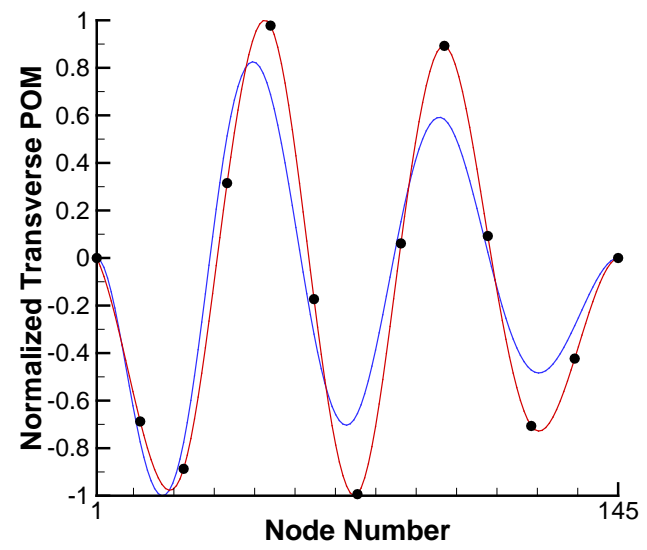

Figure 11: Transverse POM 6.

System identification data for the 11 transverse POMs obtained from acceleration data is presented in Table 1. Shown are the POV participation factors computed per Eq. (8), the cumulative POV participation factors computed per Eq. (9), the corresponding LNM (computed from the eigenanalysis of the full-order model) as determined by the MAC analysis, the LNM natural frequency, and the MAC values computed per Eq. (10). The LNM number was assigned in order of ascending natural frequency. It is seen that inaccuracies in the experimentally obtained POMs did not result in a low MAC value, nor by a need to represent a single POM with multiple LNMs.

Table 1: System identification and basis selection using transverse POVs obtained from acceleration.

\begin{tabular}{cccccc}
\hline POM & POV (\%) & Cum POV (\%) & LNM & LNM Natural Freq (Hz) & MAC \\
\hline 1 & 79.20 & 79.20 & 1 & 41.647 & 0.997 \\
2 & 16.37 & 95.58 & 2 & 104.78 & 0.919 \\
3 & 3.35 & 98.92 & 3 & 219.14 & 0.862 \\
4 & 0.61 & 99.53 & 6 & 762.42 & 0.763 \\
5 & 0.32 & 99.85 & 4 & 394.57 & 0.986 \\
6 & 0.086 & 99.94 & 5 & 587.94 & 0.941 \\
7 & 0.056 & 99.99 & 7 & 1041.8 & 0.590 \\
\hline 8 & $3.7 \mathrm{e}-4$ & 99.99 & 8 & 1395.9 & 0.910 \\
9 & $7.4 \mathrm{e}-5$ & 99.99 & 9 & 1701.8 & 0.725 \\
10 & $1.9 \mathrm{e}-5$ & 99.99 & 10 & 1919.2 & 0.770 \\
11 & $4.0 \mathrm{e}-8$ & 99.99 & 11 & 2244.1 & 0.865 \\
\hline
\end{tabular}

The selection of the transverse portion of the modal basis for the reduced order analysis was therefore based exclusively on identifying POMs above some threshold value of the cumulative POV factor. A value of $v>99.99 \%$ 
was chosen for this purpose resulting in selection of 7 POMs $\left(M_{T}\right)$ and 7 corresponding LNMs $\left(L_{T}\right)$ for the transverse portion of the modal basis, indicated by the red-line in Table 1 . The 7 selected POMs are also used to numerically determine corresponding in-plane behavior in the absence of in-plane acceleration data. Finally, it should be noted that the POD/MAC analysis using finite element displacement data identified the same 7 transverse LNMs.

\section{In-Plane Modes}

As previously indicated, two methods for selecting the in-plane LNM basis are employed. The first relies on inplane acceleration, as would be acquired with transverse acceleration using a biaxial accelerometer. In this case, the processing of in-plane acceleration to displacement time histories, and the POM/MAC analyses are identical to the approach presented in the previous section. In the more likely scenario, in-plane acceleration data is not available and the in-plane response must be derived from the transverse POMs. Both methods are next discussed.

For the case when in-plane acceleration data is available, the first 6 POMs are shown in Figure 12 - Figure 17. In each figure, the raw experimentally determined POM is shown at the nodal spacing corresponding to the accelerometer locations. The spline-fit POMs used for the MAC analyses are shown at each nodal location. For comparison, POMs obtained directly from the full-order FE analysis displacement time histories are also shown. It is seen that the set of POMs from the two methods are similar, although agreement is not as good as was observed in the transverse POMs (see Figure 6 - Figure 11).

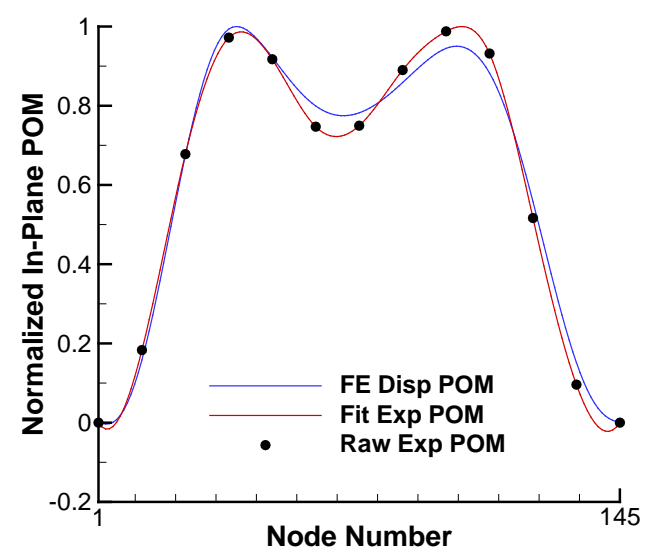

Figure 12: In-plane POM 1.

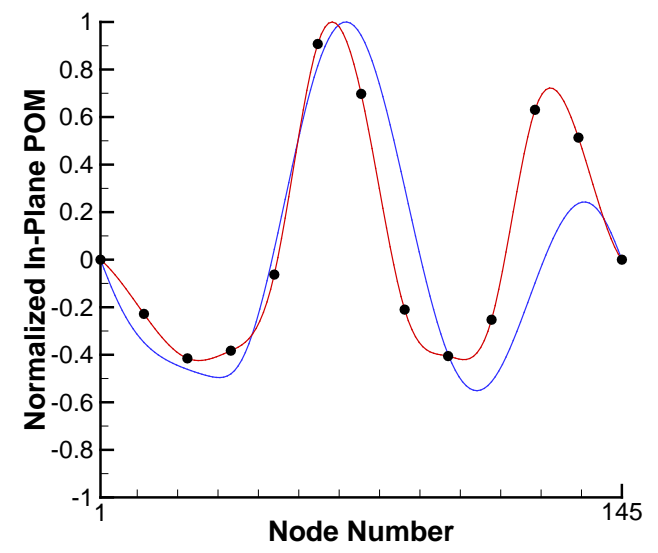

Figure 14: In-plane POM 3.

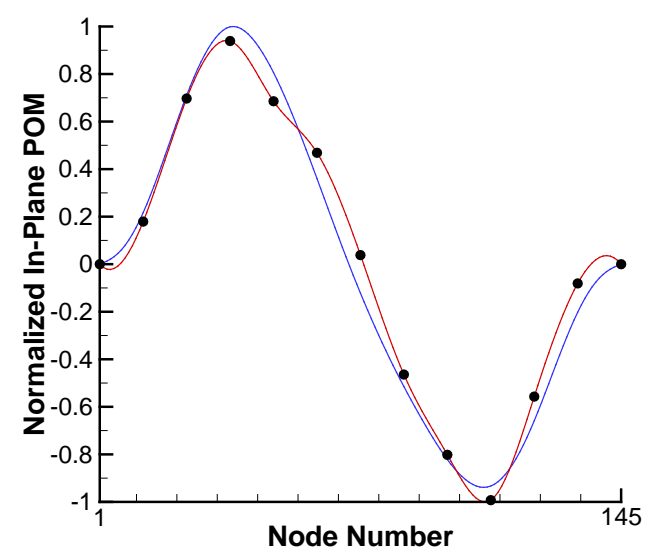

Figure 13: In-plane POM 2.

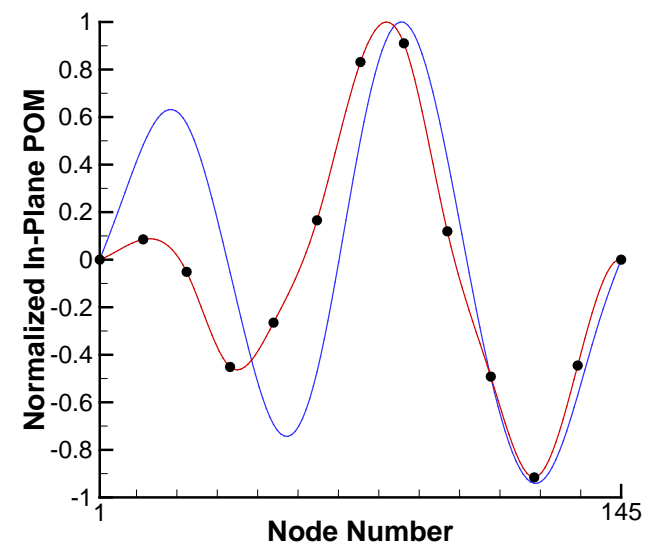

Figure 15: In-plane POM 4. 


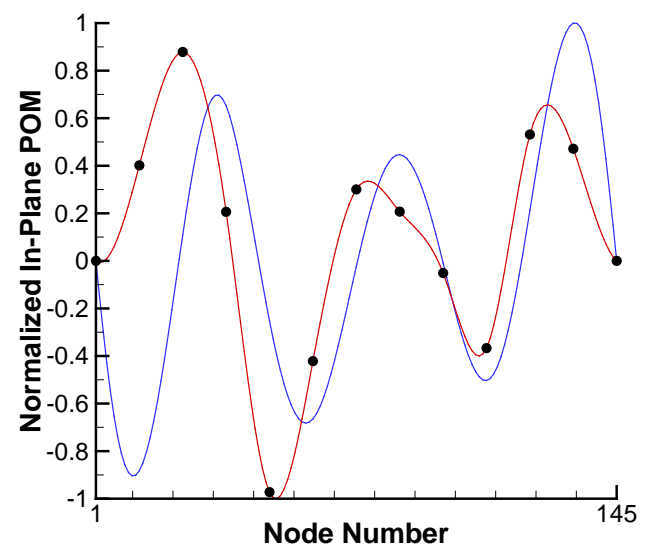

Figure 16: In-plane POM 5.

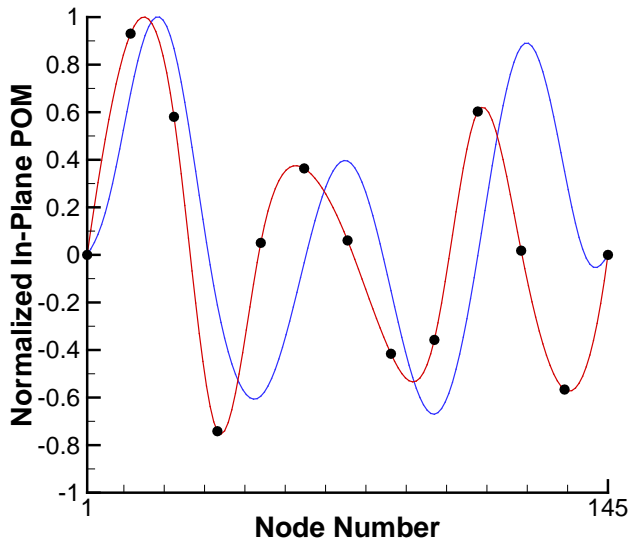

Figure 17: In-plane POM 6.

System identification data for the in-plane POMs obtained from acceleration data is presented in Table 2. It is seen that inaccuracies in the experimentally obtained POMs did result in lower MAC values compared to the transverse POMs. In nearly half the cases, the MAC criterion was unable to identify a LNM using the same cutoff value of 0.5 . As such, the cumulative POV used only reached $97.64 \%$ for the 6 LNMs $\left(L_{I}\right)$ identified. For this case, use of an alternative mapping technique, e.g. modal expansion, ${ }^{9,11,12}$ might have identified a greater number of LNMs and hence higher cumulative POV than the MAC criterion employed. While the MAC analysis using POMs obtained directly from the finite element analysis displacement time histories (not shown) was able to identify three additional LNMs (34, 39 and 69), it could not identify LNMs corresponding to POMs 9 and 10.

Table 2: System identification and basis selection using in-plane POVs obtained from acceleration.

\begin{tabular}{cccccc}
\hline POM & POV (\%) & Cum POV (\%) & LNM & LNM Natural Freq (Hz) & MAC \\
\hline 1 & 79.00 & 79.00 & 13 & 3914.9 & 0.953 \\
2 & 9.11 & 88.10 & 20 & 7411.1 & 0.796 \\
3 & 5.61 & 93.71 & 31 & 17354 & 0.508 \\
4 & 3.41 & 97.12 & 26 & 12289 & 0.642 \\
5 & 1.57 & - & - & - & $<0.5$ \\
6 & 0.65 & - & - & - & $<0.5$ \\
7 & 0.29 & 97.41 & 45 & 33492 & 0.658 \\
8 & 0.23 & 97.64 & 42 & 28671 & 0.654 \\
9 & 0.08 & - & - & - & $<0.5$ \\
10 & 0.05 & - & - & - & $<0.5$ \\
11 & 0.01 & - & - & - & $<0.5$ \\
\hline
\end{tabular}

For the more typical case when in-plane acceleration data is unavailable, the selected set of 7 experimentally determined transverse POMs were individually applied as static displacement fields in a series of nonlinear static FE analyses. The POMs were scaled to a maximum displacement of $5 \mathrm{~mm}$, or about two times the beam thickness, as this was close to the maximum transverse displacement found from the integrated acceleration data. Six of seven of the resulting displacement fields, heretofore referred to as companions, are shown in Figure 18 - Figure 23. It is clear that the companion shapes are vastly different than the POM shapes found from in-plane acceleration data. However, a linear superposition of companion modes can yield shapes similar to the in-plane POMs. It is seen that superposition of companions 1-7 reconstruct shapes similar to in-plane POMs 1 and 2, as shown in Figure 24 and Figure 25, respectively. Recall that these two POMs account for $88 \%$ of the in-plane response. Therefore, it is expected that similarities will exist between the LNMs identified using companions and those identified using the in-plane POMs.

Basis selection data for the in-plane companions is presented in Table 3. Like the in-plane POMs, MAC analyses using the in-plane companions resulted in lower MAC values compared to the transverse POMs. Because the companions were not obtained from a POD analysis, the individual and cumulative POV participation factors are not available. Therefore, the cutoff was effectively established by the cutoff used to select the 7 transverse POMs. 
A consequence of using companions instead of POMs in the MAC analyses was the identification of only three unique in-plane LNMs. However, these three LNMs are a subset of those identified using in-plane POMs, and are a reflection of the fact that companions can be superposed to reconstruct shapes similar to in-plane POMs. The effect of using only three in-plane LNMs $\left(L_{I}\right)$ on the reduced-order simulation results, versus 6 and 9 for the acceleration and displacement-based POMs, respectively, will next be discussed.

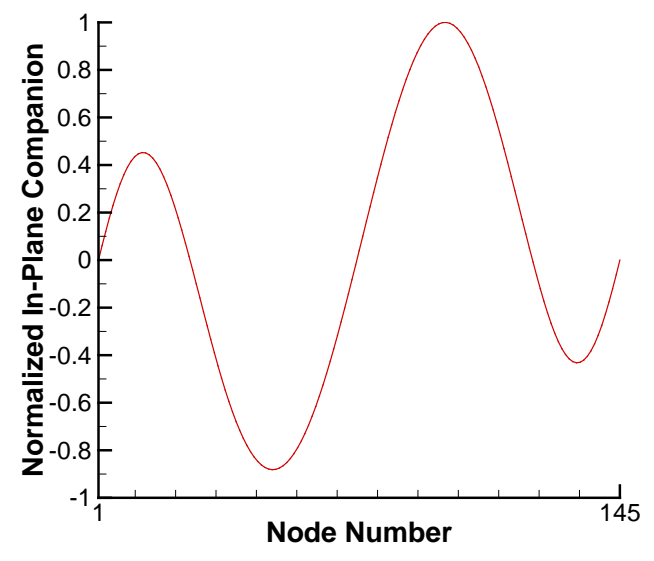

Figure 18: In-plane companion 1.

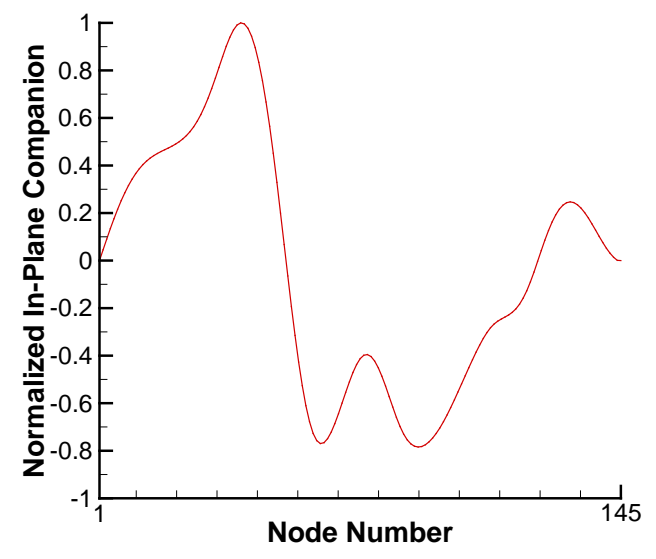

Figure 20: In-plane companion 3.

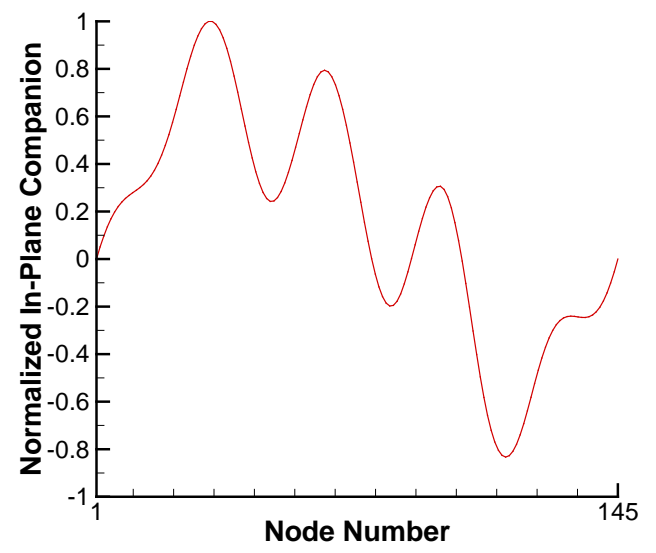

Figure 22: In-plane companion 5.

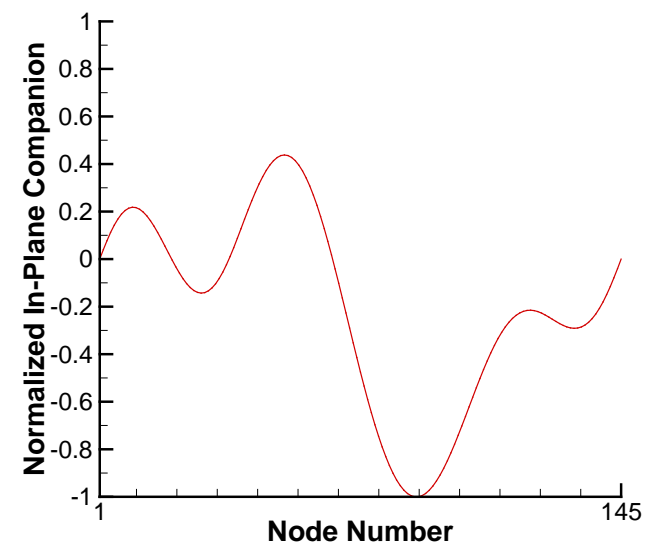

Figure 19: In-plane companion 2.

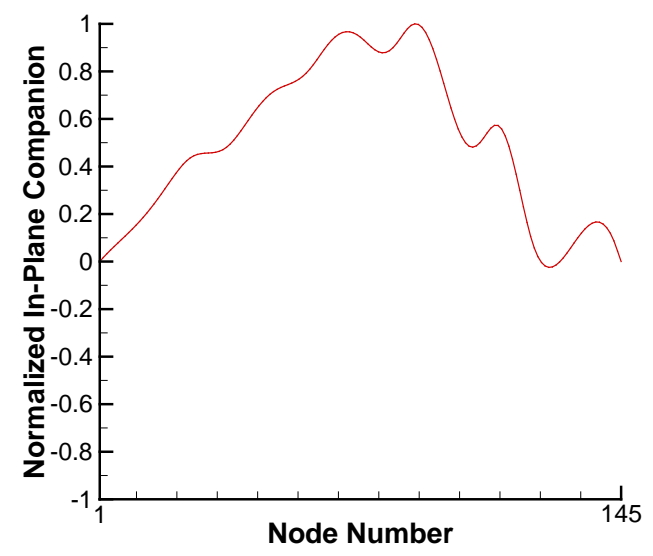

Figure 21: In-plane companion 4.

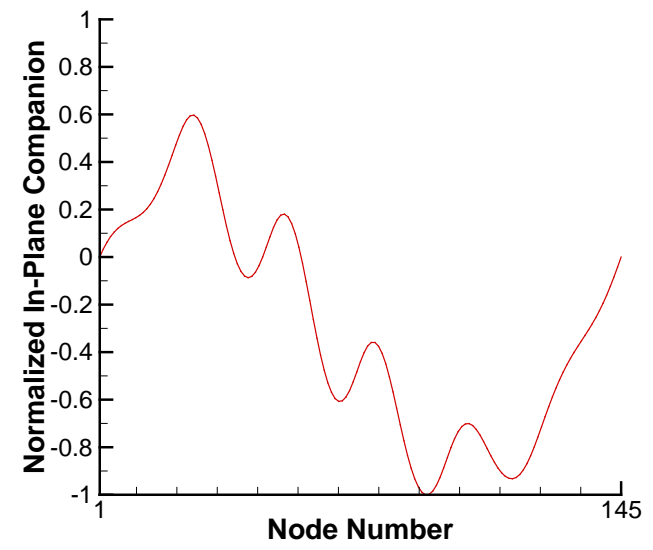

Figure 23: In-plane companion 6. 


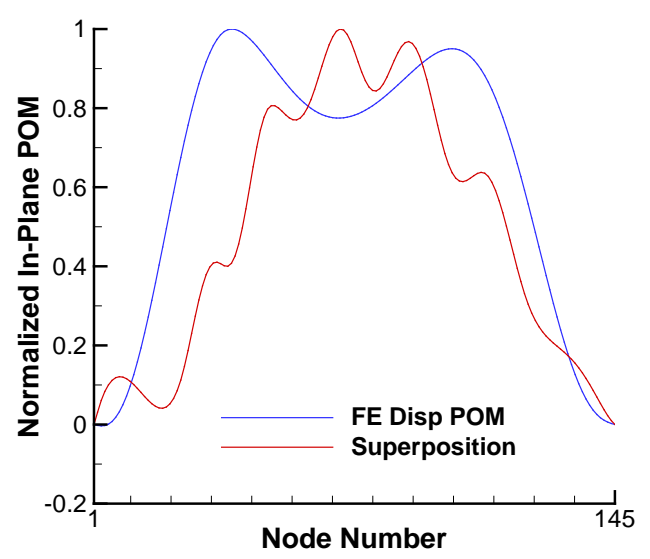

Figure 24: Superposition of companions for reconstruction of in-plane POM 1.

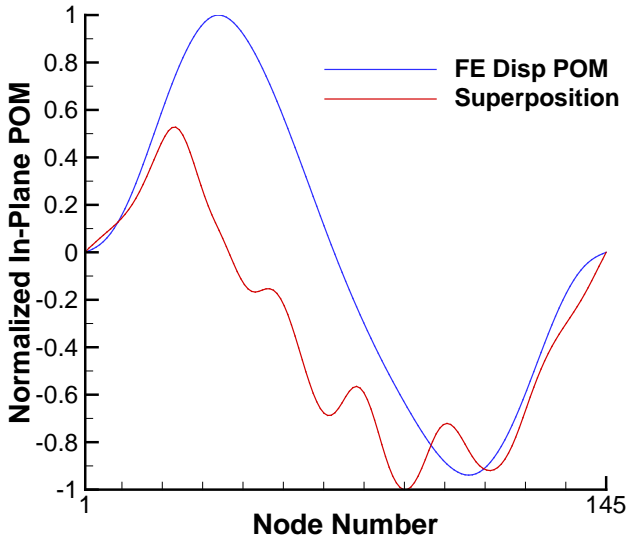

Figure 25: Superposition of companions for reconstruction of in-plane POM 2.

Table 3: Basis selection using in-plane companions obtained from transverse POMs.

\begin{tabular}{cccc}
\hline Companion & LNM & LNM Natural Freq (Hz) & MAC \\
\hline 1 & 31 & 17354 & 0.562 \\
2 & 20 & 7411.1 & 0.619 \\
3 & 20 & 7411.1 & 0.557 \\
4 & 13 & 3914.9 & 0.946 \\
5 & - & - & $<0.5$ \\
6 & 20 & 7411.1 & 0.842 \\
7 & 13 & 3914.9 & 0.901 \\
\hline
\end{tabular}

\section{Reduced-Order Simulation Results}

Four different bases were considered for the reduced-order analyses. The first consisted of only 7 transverse LNMs derived from the transverse acceleration data, and is subsequently referred to as the 7-mode acceleration derived basis. As will be shown, this basis was included to show the importance of in-plane modes and not as a desired basis. The second basis consisted of 7 transverse LNMs and 6 in-plane LNMs, each derived from acceleration data. This is subsequently referred to as the 13-mode acceleration derived basis. The third consisted of 7 transverse LNMs derived from acceleration data, and 3 in-plane LNMs derived from companions. This is subsequently referred to as the 10-mode hybrid basis. The last basis consisted of 7 transverse LNMs and 9 in-plane LNMs, each derived from displacement data. This is subsequently referred to as the 16-mode displacement derived basis. Its inclusion allows comparisons of the new acceleration derived and hybrid bases, with the previous approach of using numerical simulation data for the basis selection. Table 4 gives a breakdown of selected transverse and in-plane LNMs for each basis.

Table 4: Content of each modal basis.

\begin{tabular}{ccc}
\hline Basis & Transverse LNMs & In-Plane LNMs \\
\hline 7 mode acceleration derived & $1-7$ & none \\
10 mode hybrid basis & $1-7$ & $13,20,31$ \\
13 mode acceleration derived & $1-7$ & $13,20,26,31,42,45$ \\
16 mode displacement derived & $1-7$ & $13,20,26,31,34,39,42,45,69$ \\
\hline
\end{tabular}

A comparison of the transverse displacement PSDs at the drive point is shown in Figure 26. All reduced-order analyses compare well with the simulated experimental data, with differences noted at the higher frequencies due to modal truncation. Because of nonlinear coupling, peaks in the response are seen outside of the excitation bandwidth. To highlight the differences between the reduced-order analyses, a close-up of the low frequency response is shown in Figure 27. There it is seen that a lesser number of in-plane modes in the basis (recall that all reduced-order analyses have the same 7 transverse modes) acts to over-predict the degree of nonlinear springhardening. The 7-mode acceleration derived basis, with no in-plane modes, shows the most spring-hardening as 
indicated by a shifting of peaks to higher frequencies, a greater degree of peak broadening, and reduced amplitude relative to the experiment. Addition of in-plane modes lessens the degree of nonlinear spring-hardening, with the 13-mode acceleration derived basis and 16-mode displacement derived basis virtually indistinguishable from the experiment.

The in-plane displacement PSDs at the drive point are shown in Figure 28, and in close-up view in Figure 29. All three reduced-order analyses compare favorably with the experiment at low frequencies and follow similar trends at higher frequencies. It should be noted that the behavior shown is solely due to coupling between the transverse and in-plane modes, not direct excitation of the in-plane modes which are at higher frequencies (see Table 2 and Table 3). The 7-mode acceleration derived basis is incapable of predicting any in-plane response since it does not contain any in-plane modes. The close-up view indicates that the reduced-order analyses improve by increasing the number of modes, as expected.

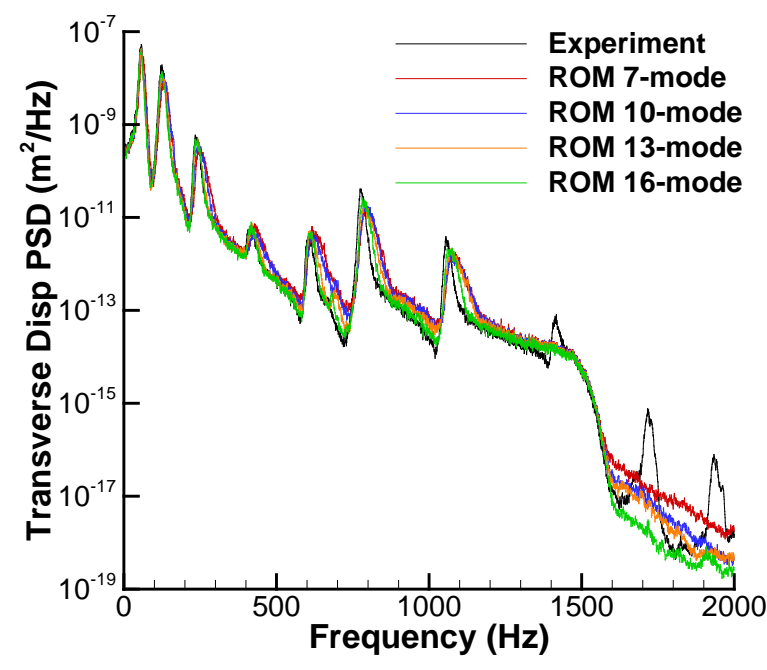

Figure 26: Comparison of transverse drive point displacement PSD for the $12.8 \mathrm{~N}$ RMS loading.

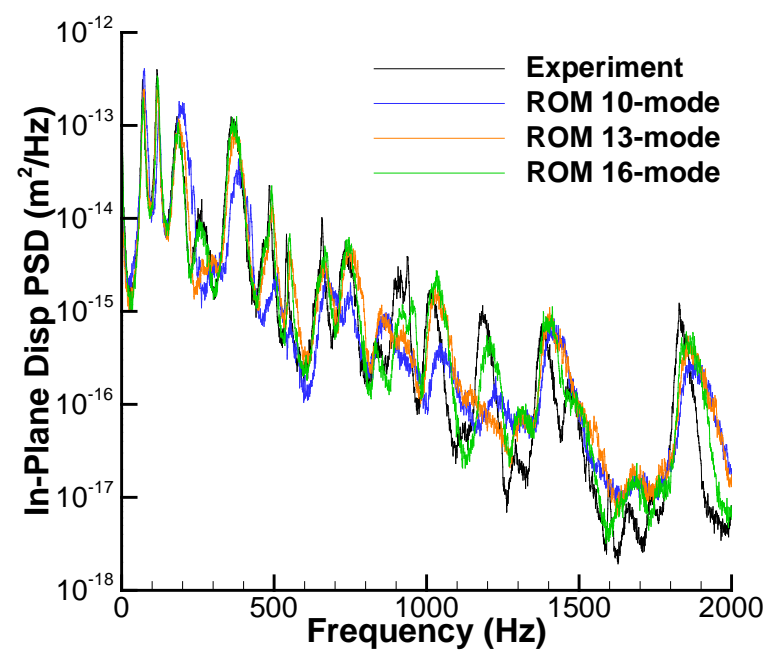

Figure 28: Comparison of in-plane drive point displacement PSD for the 12.8N RMS loading.

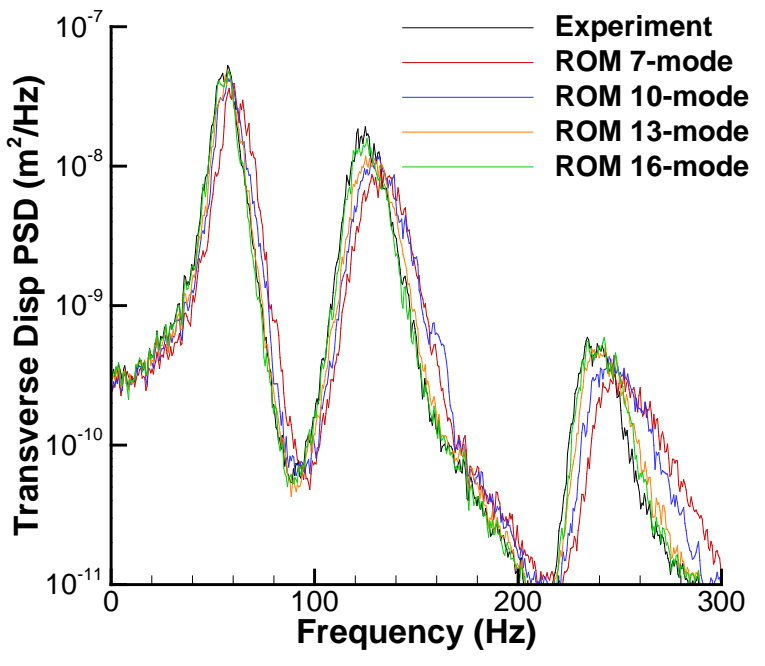

Figure 27: Close-up of transverse drive point displacement PSD for the 12.8N RMS loading.

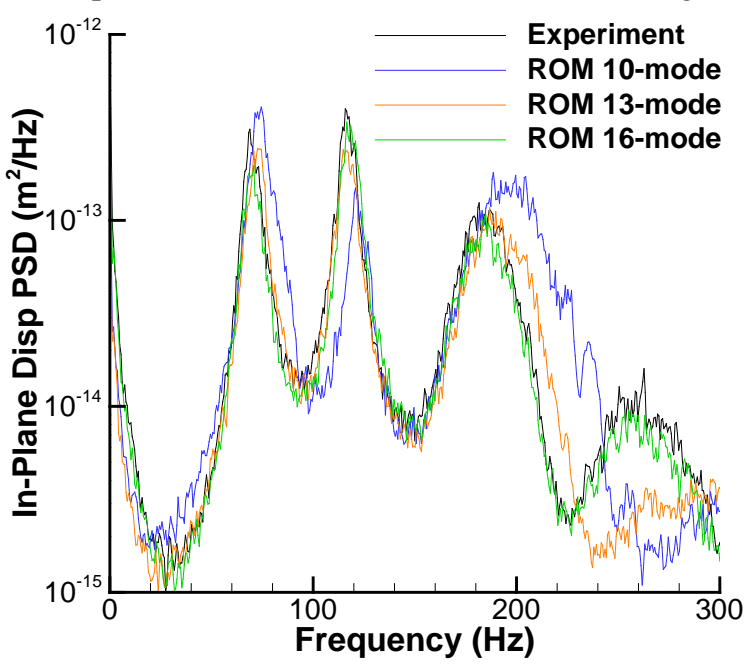

Figure 29: Close-up of in-plane drive point displacement PSD for the 12.8N RMS loading.

Finally, recall that the basis consisting of load-independent LNMs, instead of one consisting of load-dependent POMs, was selected in order to form a robust basis applicable over a range of loadings. This is demonstrated in Figure 30 and Figure 31 for a far lower excitation of 0.1N RMS, where excellent comparison of all reduced-order analyses with the simulated experimental data is seen across the frequency range. Again, exceptions are noted at high frequency due to modal truncation, and in the 7-mode basis for the in-plane response. 
The conclusion for this idealized case is that reduced-order models formed with LNM bases identified from a system identification approach using either both transverse and in-plane acceleration data, or only transverse acceleration data, are a viable alternative to bases identified with the previous displacement based approach. Of the two approaches, the basis selected using both transverse and in-plane acceleration data was more accurate as it was able to identify a greater number of in-plane modes. Next investigated is the non-idealized case where modeling uncertainty in the boundary conditions and accelerometer mass loading are considered to more closely mimic actual experimental conditions.

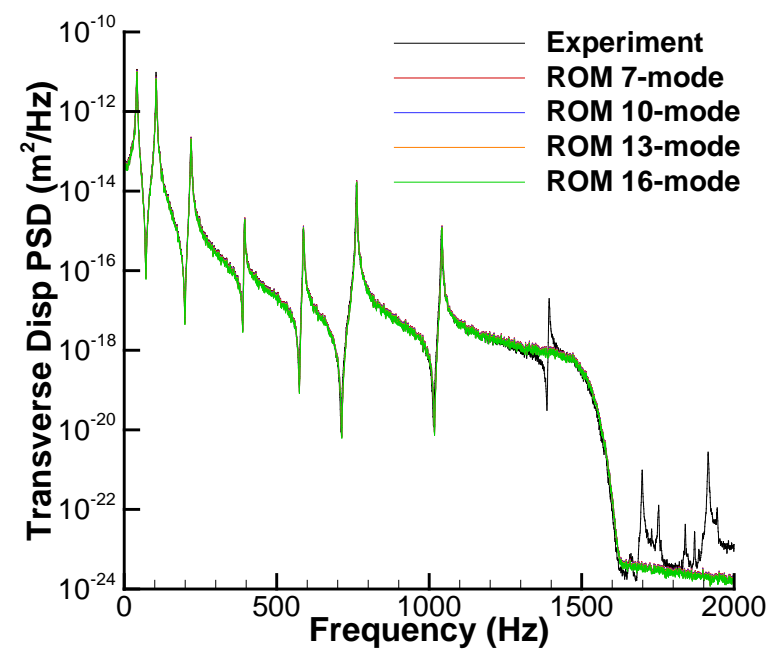

Figure 30: Comparison of transverse drive point displacement PSD for the 0.1N RMS loading.

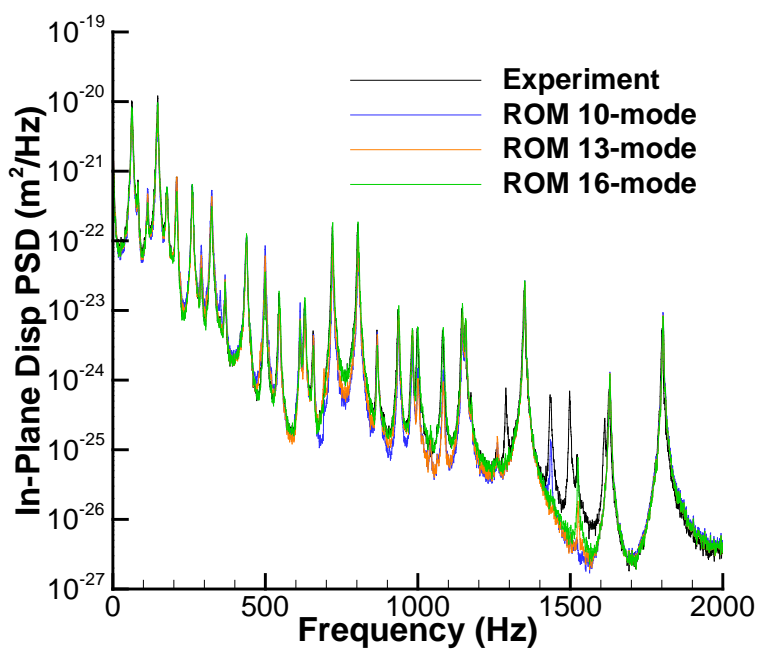

Figure 31: Comparison of in-plane drive point displacement PSD for the 0.1N RMS loading.

\section{Non-Idealized Case}

The non-idealized case utilized the same beam structure as the idealized case, but with compliant boundary conditions and variations in the accelerometer mass distribution. The same transverse random loading was applied at the $1 / 4$-span location. System identification was performed at the $12.8 \mathrm{~N}$ RMS loading level. The basis used in the reduced-order model was developed from the system identification using transverse acceleration data alone, i.e. the hybrid basis, since in-plane acceleration data is not typically available.

\section{A. Surrogate Experiment}

Compliant boundary conditions were simulated using two node CONN2D2 connector elements in ABAQUS. ${ }^{18}$ The in-plane and transverse spring stiffness were set high at $10 \mathrm{GN} / \mathrm{m}$ to simulated immovable boundaries. The rotational stiffness was set at $500 \mathrm{~N}-\mathrm{m}$ on the left hand side, and $100 \mathrm{~N}-\mathrm{m}$ on the right hand side.

The mass loading of the accelerometers was altered in both magnitude and distribution. The magnitude was changed to account for varying lead wire mass by allowing the total mass of each accelerometer to vary between 0.4-0.6g. This increased the total added mass from $44 \mathrm{~g}$ in the idealized case to 55.62g for the non-idealized case. The accelerometers were assumed to have a mounted base dimension of $5.8 \mathrm{~mm}$. They therefore span two elements or 3 nodes in the surrogate experiment finite element model. The randomized mass was itself randomly distributed across the 3 nodes to allow the center mounting location to deviate from the nominal location by as much as $1.6 \mathrm{~mm}$. The load cell was assumed to have a mounted base dimension of $16 \mathrm{~mm}$ and its mass was distributed equally across 5 nodes. The total mass of $25 \mathrm{~g}$ was left unchanged from the idealized case.

The net effect of these changes was a $12 \%$ reduction in the fundamental frequency from $41.65 \mathrm{~Hz}$ to $36.72 \mathrm{~Hz}$, and asymmetry of the LNM shapes (not shown) due to different compliance at the left and right boundaries. Note that the compliant boundary conditions result in a fundamental frequency much closer to an idealized clamped condition than to an idealized simply supported condition $(17.22 \mathrm{~Hz})$.

\section{B. Full-Order Model Calibration}

Before embarking on the reduced-order model, it was first necessary to calibrate the full-order model from which the ROM is derived. This was not necessary for the idealized case because the full-order model was the same as the surrogate experimental FE model. Here, the intention is to calibrate the full-order model in a manner that might be 
performed by the experimental analyst. In this case, the calibration method is focused on matching the fundamental frequency, without regard to the mode shape. In this process, material properties of the beam were assumed to be well known and were not altered.

A static deflection test was first performed to calibrate the boundary stiffness. Via simulated experiment, a static transverse load of $1.0 \mathrm{~N}$ was applied to the surrogate experimental FE model at the 1/4-span shaker location and a transverse deflection of $0.126 \mathrm{~mm}$ was read using a linear variable differential transformer (LVDT). The rotational stiffness of the CONN2D2 elements in the full-order model were adjusted to equal values until the simulated LVDT measurement matched that of the experiment. No attempt was made to use mode shapes to make a differential adjustment. Ultimately, a rotational stiffness of $361.94 \mathrm{~N}-\mathrm{m}$ was equally applied to the left and right sides.

A dynamic test was next performed to calibrate the effect of mass. In the full-order model, all masses were applied in an idealized fashion, i.e. at a single node. Only the mass of the accelerometers was adjusted to match the fundamental frequency, and with the same adjustment applied to all masses. It was determined that an accelerometer mass of $0.61 \mathrm{~g}$ was required to match the fundamental frequency.

The accelerance frequency response functions of the experiment and calibrated full-order models are shown at the drive point and center span locations in Figure 32 and Figure 33, respectively. The calibrated full-order model matches the experimental data very well below $300 \mathrm{~Hz}$. At higher frequencies, the softness of the full-order model relative to the experiment becomes more apparent. Since the reduced-order model is derived from the calibrated full-order model, similar behavior is expected.

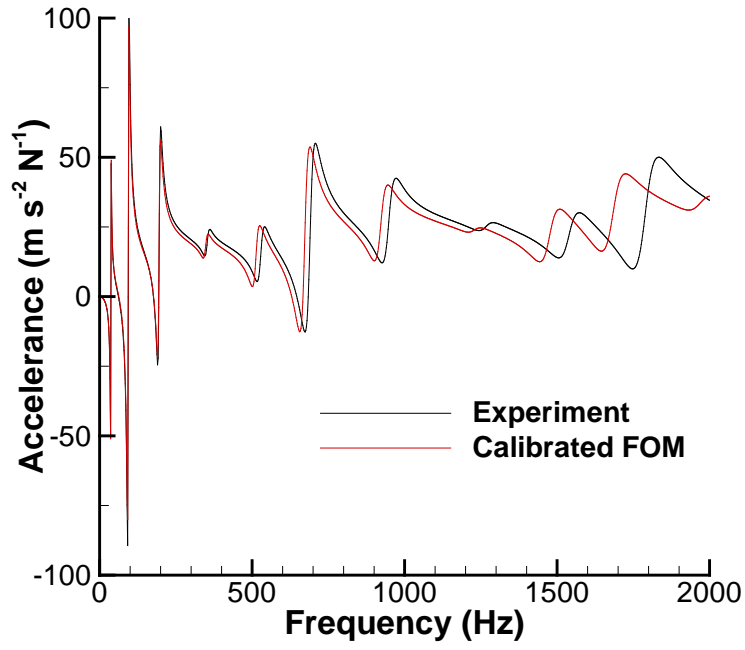

Figure 32: Comparison of transverse accelerance at drive point.

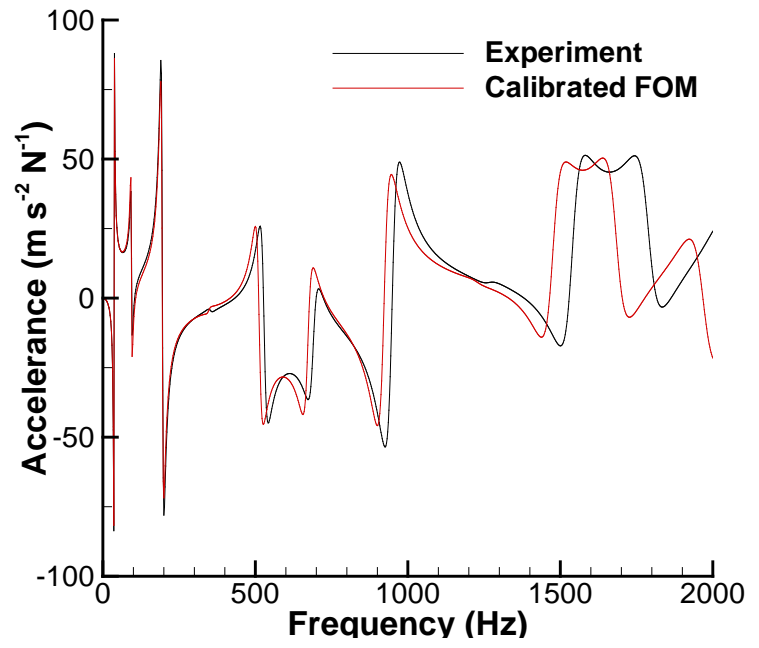

Figure 33: Comparison of transverse accelerance at center span.

\section{System Identification and Modal Basis Selection}

The hybrid approach using transverse acceleration data and companions for the in-plane response yielded a modal basis consisting of 7 transverse modes and 2 in-plane modes, as indicated in Table 5 . Like the results in Table 3 for the idealized case, the same LNMs were identified with multiple companions and the MAC values were generally low.

\section{Reduced-Order Simulation Results}

A comparison of results from the calibrated reduced-order model and the experimental are shown in Figure 34 Figure 37. For reference, also shown are the simulated responses from the calibrated full-order model. These represent the best any reduced-order model can be expected to perform. As would be expected from the linear accelerance in Figure 32, the full-order and reduced-order analyses compare most favorably at low frequencies and differ with increasing frequency. From the close-ups in Figure 35 and Figure 37, the calibrated full-order model is seen to compare very well with the experiment. Like the idealized case, the reduced order model indicates somewhat greater nonlinearity, with a quality comparable to that of the 10-mode basis for the idealized case. Therefore, it may be concluded that modeling uncertainties do not affect the reduced-order model results any more than the full-order model from which it was derived. 
Table 5: System identification and basis selection results using the hybrid approach.

\begin{tabular}{cccccc}
\hline POM/Companion & POV (\%) & Cum POV (\%) & LNM & LNM Natural Freq (Hz) & MAC \\
\hline POM 1 & 72.18 & 72.18 & 1 & 36.721 & 0.980 \\
POM 2 & 22.77 & 94.95 & 2 & 93.738 & 0.882 \\
POM 3 & 3.45 & 98.40 & 3 & 194.68 & 0.915 \\
POM 4 & 0.77 & 99.17 & 4 & 347.35 & 0.843 \\
POM 5 & 0.55 & 99.73 & 6 & 672.27 & 0.688 \\
POM 6 & 0.22 & 99.95 & 5 & 512.54 & 0.731 \\
POM 7 & 0.04 & 99.99 & 7 & 922.03 & 0.785 \\
\hline Companion 1 & n/a & n/a & 12 & 3623.6 & 0.529 \\
Companion 2 & n/a & n/a & 20 & 6869.5 & 0.550 \\
Companion 3 & n/a & n/a & 12 & 3623.6 & 0.939 \\
Companion 4 & n/a & n/a & 12 & 3623.6 & 0.792 \\
Companion 5 & n/a & n/a & 12 & 3623.6 & 0.755 \\
Companion 6 & n/a & n/a & 12 & 3623.6 & 0.737 \\
Companion 7 & n/a & n/a & 12,20 & $3623.6,6869.5$ & $0.516,0.585$ \\
\hline
\end{tabular}

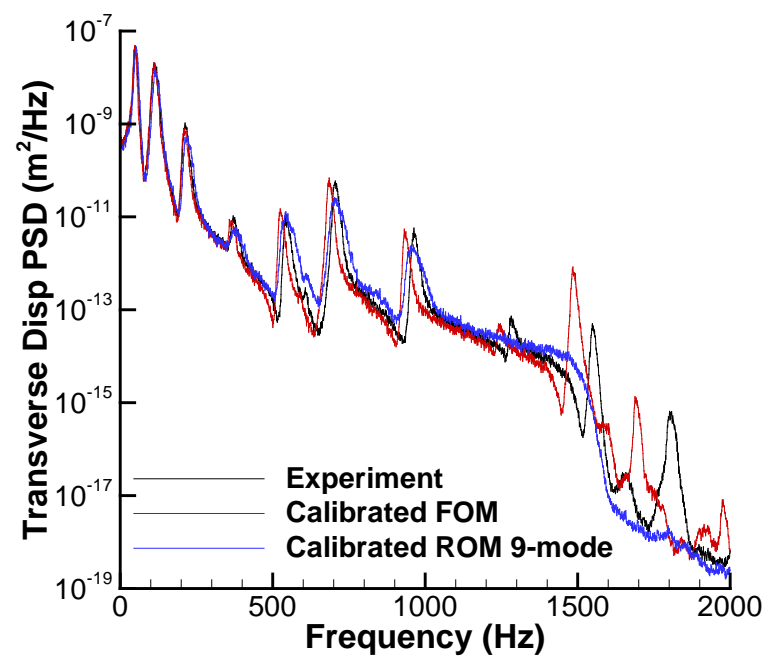

Figure 34: Comparison of transverse drive point displacement PSD for the 12.8N RMS loading.

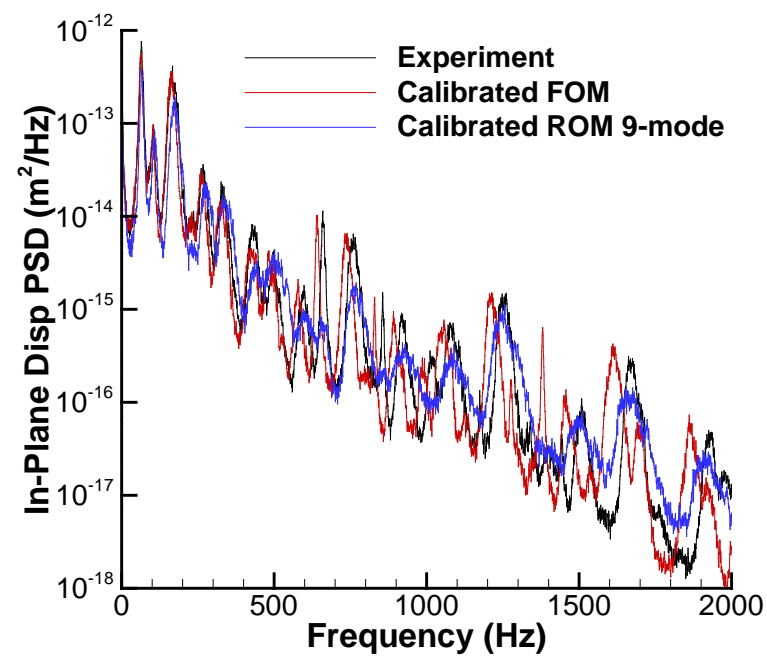

Figure 36: Comparison of in-plane drive point displacement PSD for the 12.8N RMS loading.

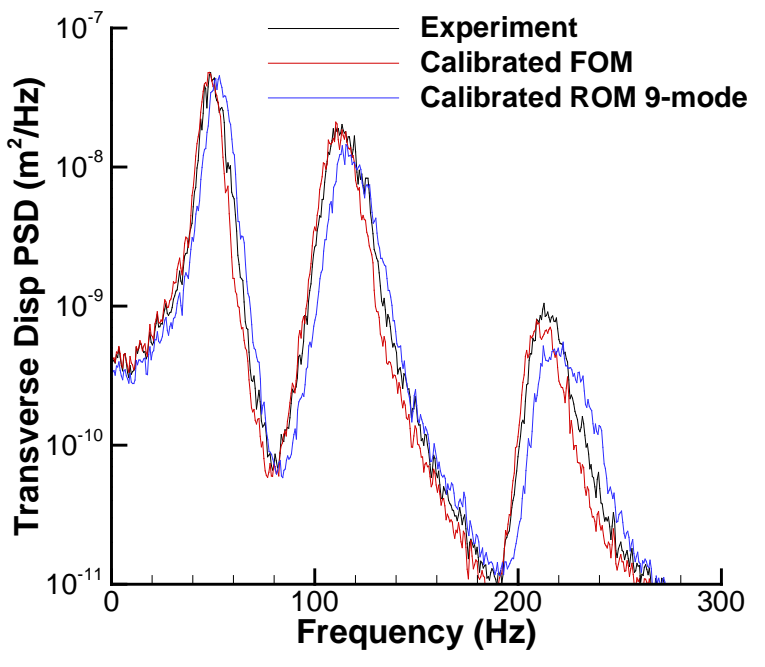

Figure 35: Close-up of transverse drive point displacement PSD for the 12.8N RMS loading.

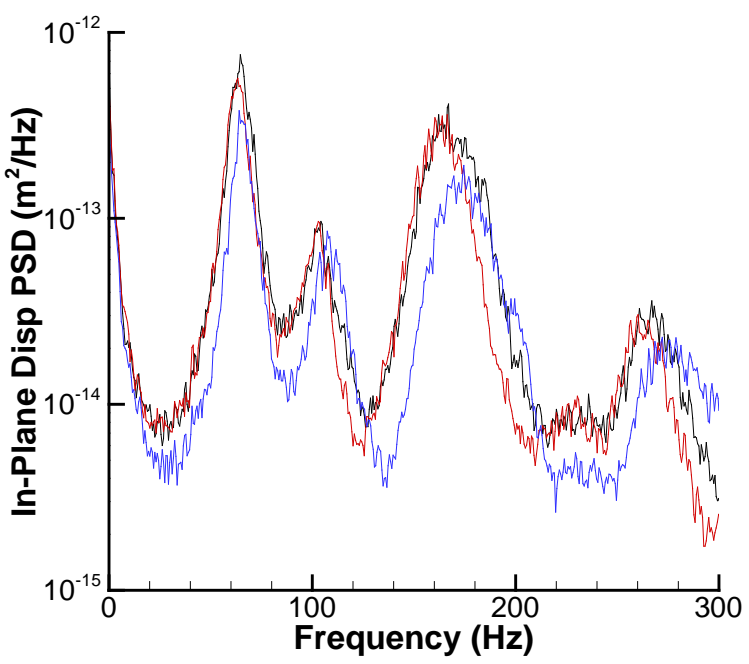

Figure 37: Close-up of in-plane drive point displacement PSD for the 12.8N RMS loading.

American Institute of Aeronautics and Astronautics 


\section{Concluding Remarks}

An approach has been developed to utilize experimental acceleration data to guide the modal basis selection for a nonlinear reduced-order simulation. Two variations were explored; one in which only the transverse acceleration data was used, and one in which both transverse and in-plane acceleration data were used. In the former, a hybrid of transverse acceleration derived POMs and in-plane companions were used to select the modal basis, while in the latter transverse and in-plane acceleration derived POMs were used. Both variations were able to identify bases which resulted in favorable reduced-order simulations relative to the simulated experiment, and relative to reducedorder simulations whose bases were determined from the earlier approach using the simulated displacement response in the system identification process. Results using both transverse and in-plane accelerations in the basis selection yielded higher quality simulations than the hybrid approach because a greater number of in-plane modes were selected through the system identification process. Use of modal expansion instead of MAC for in-plane basis selection could benefit both in-plane POMs and companions as both were distorted relative to the displacement derived POMs.

The approach was demonstrated for an idealized case in which all modeling parameters were known, and a more realistic case in which modeling uncertainties associated with boundary conditions and added mass were included. It was found that these modeling uncertainties did not negatively affect the reduced-order model results any more than the full-order model from which it was derived. Through this work, the groundwork has been established to permit the use of actual experimental data for basis selection in future studies.

\section{Acknowledgments}

The authors wish to thank Alexander Shaw, University of Bristol, Advanced Composites Centre for Innovation and Science (ACCIS), and Dr. Alessandro Carrella, LMS International nv, for helpful discussions and future test planning.

\section{References}

${ }^{1}$ Muravyov, A.A. and Rizzi, S.A., "Determination of nonlinear stiffness with application to random vibration of geometrically nonlinear structures," Computers and Structures, Vol. 81, No. 15, 2003, pp. 1513-1523.

${ }^{2}$ Mei, C., "Three decades' interesting experience in nonlinear finite element formulation development and aerospace applications," Structural Dynamics: Recent Advances, Proceedings of the 8th International Conference, Paper No. 1, Southampton, UK, 2003, The Institute of Sound and Vibration Research, University of Southampton.

${ }^{3}$ Hollkamp, J.J., Gordon, R.W., and Spottswood, S.M., "Nonlinear modal models for sonic fatigue response prediction: a comparison of methods," Journal of Sound and Vibration, Vol. 284, No. 3-5, 2005, pp. 1145-1163.

${ }^{4}$ Mignolet, M.P., Radu, A.G., and Gao, X., "Validation of reduced order modeling for the prediction of the response and fatigue life of panels subjected to thermo-acoustic effects," Structural Dynamics: Recent Advances, Proceedings of the 8th International Conference, Paper No. 55, Southampton, UK, 2003, M.J. Brennan, et al. (ed.), The Institute of Sound and Vibration Research, University of Southampton.

${ }^{5}$ McEwan, M.I., Wright, J.R., Cooper, J.E., and Leung, Y.T., "A combined modal/finite element analysis technique for the dynamic response of a non-linear beam to harmonic excitation," Journal of Sound and Vibration, Vol. 243, No. 4, 2001, pp. 601-624.

${ }^{6}$ Dhainaut, J.M., Duan, B., Mei, C., Spottswood, S.M., and Wolfe, H.F., "Non-linear response of composite panels to random excitations at elevated temperatures," Structural Dynamics: Recent Advances, Proceedings of the 7th International Conference, Vol. 2, Southampton, England, 2000, pp. 769-784.

${ }^{7}$ Tiso, P., Jansen, E., and Abdalla, M., "A reduction method for finite element nonlinear dynamic analysis of shells," Proceedings of the 47th AIAA/ASME/ASCE/AHS/ASC Structures, Structural Dynamics, and Materials Conference, AIAA-20061746, Newport, RI, 2006.

${ }^{8}$ Guo, X., Lee, Y.Y., and Mei, C., "Nonlinear random response of laminated composite shallow shells using finite element method," International Journal for Numerical Methods in Engineering, Vol. 67, No. 10, 2006, pp. 1467-1489.

${ }^{9}$ Przekop, A., Guo, X., and Rizzi, S.A., "Alternative modal basis selection procedures for nonlinear random response simulation," Structural Dynamics: Recent Advances, Proceedings of the 10th International Conference, Paper 36, Southampton, UK, 2010, M.J. Brennan, et al. (ed.).

${ }^{10}$ Spottswood, S.M. and Allemang, R.J., "On the investigation of some parameter identification and experimental modal filtering issues for nonlinear reduced order models," Experimental Mechanics, Vol. 47, No. 2007, pp. 511-521.

${ }^{11}$ Przekop, A. and Rizzi, S.A., "An efficient modal basis selection criteria for reduced-order nonlinear simulation," Proceedings of the 7th European Conference on Structural Dynamics, Paper E69, Southampton, UK, 2008.

${ }^{12}$ Przekop, A. and Rizzi, S.A., "Nonlinear reduced-order analysis with time-varying spatial loading distributions," Journal of Aircraft, Vol. 46, No. 4, 2009, pp. 1395-1402.

${ }^{13}$ Rizzi, S.A. and Przekop, A., "System identification-guided basis selection for reduced-order nonlinear response analysis," Journal of Sound and Vibration, Vol. 315, No. 3, 2008, pp. 467-485. 
${ }^{14}$ Rizzi, S.A. and Przekop, A., "Estimation of sonic fatigue by reduced-order finite element based analysis," Structural Dynamics: Recent Advances, Proceedings of the 9th International Conference, Paper 129, Southampton, UK, 2006, M.J. Brennan, et al. (ed.), The Institute of Sound and Vibration Research, University of Southampton.

${ }^{15}$ Feeny, B.F. and Kappagantu, R., "On the physical interpretation of proper orthogonal modes in vibration," Journal of Sound and Vibration, Vol. 211, No. 4, 1998, pp. 607-616.

${ }^{16}$ Kumar, N. and Burton, T.D., "Use of random excitation to develop POD based reduced order models for nonlinear structural dynamics," Proceedings of the ASME 2007 International Design Engineering Technical Conference and Information in Engineering Conference IDETC/CIE 2007, DETC2007-35539, Las Vegas, NV, 2007.

${ }^{17}$ Allemang, R.J. and Brown, D.L., "A correlation coefficient for modal vector analysis," Proceedings of the International Modal Analysis Conference, 1982, pp. 110-116.

${ }^{18}$ ABAQUS 6.10-EF Online Documentation. Dassault Systemes Simulia Corp., Providence, RI, 2010.

${ }^{19}$ MATLAB R2010b Online Documentation. The MathWorks, Inc., Natick, MA, 2011. 